\title{
First experimental demonstration of forced dynamic aperture measurements with LHC ac dipoles
}

\author{
F. S. Carlier \\ CERN, Geneva CH-1211, Switzerland and Nikhef, Amsterdam 1098 XG, Netherlands \\ R. Tomás, E. H. Maclean, and T. H. B. Persson \\ CERN, Geneva CH-1211, Switzerland
}

(Received 17 August 2018; published 13 March 2019)

\begin{abstract}
Diagnostics of nonlinear beam dynamics has become more important for the LHC as it advances into increasingly challenging regimes of operation, as well as for the High Luminosity LHC where machine nonlinearities will have a significantly larger impact. Limitations of traditional excitation methods at top energy, in particular due to machine protection, have pushed the development of safe alternative methods using ac dipoles to characterize the nonlinear content of the LHC. One of the methods that has been proposed is the dynamic aperture under forced oscillation of ac dipoles. This new observable has the potential to help characterize relative changes in the nonlinear content of the machine, improve the understanding of the nonlinear models by comparing to simulations, validate nonlinear optics corrections, and give a qualitative lower bound estimate on the free dynamic aperture. This paper presents the first experimental demonstration of forced dynamic aperture measurements under forced oscillations performed using the LHC ac dipoles, and discusses the benefits of forced dynamic aperture measurements in circular colliders.
\end{abstract}

DOI: $10.1103 /$ PhysRevAccelBeams.22.031002

\section{INTRODUCTION}

The ac dipole system is able to generate coherent transverse beam oscillations. It was first developed for synchrotrons to avoid spin resonances in the AGS [1] and later used for the first time to measure linear optics functions in RHIC [2-4] and the Tevatron [5-7]. ac dipoles were proposed in the LHC as a complementary tool to measure optics functions alongside the more conventional kickers (MKQ and MKA $[8,9])$. However over the years, the importance of the LHC ac dipoles $[10,11]$ has grown and they have now become critical in correcting linear [12-14] and nonlinear [15-17] optics parameters. To such an extent that they are the single most important measurement method for the linear and nonlinear correction strategies in the LHC and for the High Luminosity LHC [18]. This is in part due to the fact that single kicks are not allowed at top energy for machine protection considerations.

The success and reliability of ac dipoles have allowed them to be used for new applications. The LHC ac dipoles have been used to measure the physical aperture of the

\footnotetext{
*felix.simon.carlier@cern.ch

Published by the American Physical Society under the terms of the Creative Commons Attribution 4.0 International license. Further distribution of this work must maintain attribution to the author(s) and the published article's title, journal citation, and DOI.
}

collider to assist in collimator alignment [19], to measure amplitude detuning in the presence of head-on beam-beam interactions [20] as well as to measure the machine impedance [21]. More recently ac dipoles have been used in lightsources, such as at ESRF [22], PETRA III [23] and ALBA [24]. Furthermore, ac dipoles are currently foreseen for the AGS to compensate spin resonances for the eRHIC project [25] for the transport of polarized helions.

It is clear that functionality and importance of ac dipoles have grown significantly. At the core of this success lies the fact that the ac dipole can generate safe coherent transverse beam oscillation while recovering the original beam after the excitation. The slow adiabatic ramp up of the ac dipole current allows for a continuous monitoring of losses and enough time to allow safe dump triggers at top energy in the LHC. Second, the slow adiabatic ramp down of the ac dipole current makes sure the beam emittance is recovered after excitations, which means that multiple excitations can be performed in series thus drastically cutting down measurement time.

Similarly to particles in free motion, stable particles under forced oscillations are confined to a region in phase space. Beyond the boundary of stable motion in phase space particles are lost. This stability region under forced oscillations is called the forced dynamic aperture [26] and it is analogous to the dynamic aperture for free motion [27-30] which is defined here as free dynamic aperture. It is important to note that the forced dynamic aperture is a 
different physical quantity than the free dynamic aperture. Indeed a direct scaling law between free and forced dynamic apertures may not be trivially found for synchrotrons.

A poor forced dynamic aperture can significantly affect commissioning strategies with ac dipoles for highly nonlinear machines, for example for the High Luminosity LHC [18]. It is thus desirable to maximize the forced dynamic aperture to facilitate linear and nonlinear optics commissioning with ac dipoles. Furthermore, the forced dynamic aperture can be generated by any coherent harmonic excitations and is thus of particular interest to understand the effect of induced forced oscillations coming from imperfect power supplies or possible harmonic excitations from crab cavities [31]. Such sources of forced motion may significantly affect machine performance, and motivate a good understanding of forced oscillations in hadron colliders.

Fortunately, forced dynamic aperture does not only bring limitations. It is also a new alternative observable which has the potential to provide fast characterization of the nonlinear content of the machine, improve the understanding of the nonlinear models by comparing to simulations, and validate nonlinear optics corrections. It is viewed as an important complementary figure of merit for nonlinear optics commissioning strategies in the LHC and High Luminosity LHC [18].

In this paper we describe the first experimental demonstration of forced dynamic aperture measurements. The LHC ac dipoles are limited to an excitation of 10000 turns while typical free dynamic aperture studies consider measurements lasting above an order of magnitude longer. The studies in this paper are therefore restricted to short term forced dynamic aperture though the concepts are applicable to longer timescales as well. This paper proceeds by describing the influence of an ac dipole on the forced dynamic aperture in Sec. II. This is followed by results obtained through MAD-X [32] single particle tracking simulations in Sec. III where the effect of the ac dipole driving tunes on the forced dynamic aperture is explored. In Sec. IV the forced dynamic aperture is defined from beam intensity losses. Finally, a first demonstration of forced dynamic aperture measurements at injection energy in the LHC is presented in Sec. V.

\section{INFLUENCE OF AC DIPOLE ON FORCED DYNAMIC APERTURE}

Particle dynamics under the driven motion of an ac dipole are considerably altered $[7,15,17]$. The general solution for the driven particle motion with an ac dipole can be expressed at any longitudinal location $s$ of the ring as a function of turn number $T$ as

$$
\begin{aligned}
\hat{z}(T)-i \hat{p}_{z}(T)= & \sqrt{2 J_{z}} e^{i\left(2 \pi Q_{z} T+\phi_{z 0}\right)} \\
& +e^{-i \phi_{z}^{a c}}\left(\delta_{z,-} e^{i 2 \pi Q_{z}^{a c} T}-\delta_{z,+} e^{-i 2 \pi Q_{z}^{a c} T}\right),
\end{aligned}
$$

where $z \in\{x, y\}$ denotes the plane of motion, $Q_{z}$ is the betatron tune, $Q_{z}^{\mathrm{ac}}$ is the ac dipole driving tune, $\phi_{z}^{\mathrm{ac}}$ is the phase advance between the location $s$ and the ac dipole, $J$ and $\phi_{z 0}$ are the initial action and phase of the particle, and finally $\delta_{z,-}$ and $\delta_{z,+}$ are the complex ac dipole strengths defined as

$$
\delta_{z, \pm}=\sqrt{\beta_{z}^{a c}} \frac{B L}{B_{0} \rho} \frac{e^{ \pm i\left(\pi\left(Q_{z}^{a c} \pm Q_{z}\right)-\psi_{0}\right)}}{4 \sin \left(\pi\left(Q_{z}^{a c} \pm Q_{z}\right)\right)}
$$

where $\beta_{z}^{a c}$ is the $\beta$-function at the location of the ac dipole. Generally the ac dipole tunes are chosen close to the natural tunes which leads to $\left|\delta_{z,-}\right| \gg\left|\delta_{z,+}\right|$.

In the case of free oscillations, where $\delta_{z, \pm}=0$, the resonance condition obtained from the normal form formalism [33], is conventionally given by

$$
\begin{aligned}
& (j-k) Q_{x}+(l-m) Q_{y}=p \\
& \text { with, } \quad p \in \mathbb{Z} \text { and } j, k, m, l \in \mathbb{N}_{0},
\end{aligned}
$$

where $j, k, l$, and $m$ are the indices of the Hamiltonian term $h_{j k l m}$. The Hamiltonian terms are defined as in [33] as the sum over the individual sources along the accelerator,

$$
h_{j k l m}=\sum_{w} h_{w, j k l m} e^{i\left[(j-k) \Delta \phi_{x}+(l-m) \Delta \phi_{y}\right]}
$$

where $w$ is the location of a multipolar source, and $\Delta \phi_{x, y}$ are the phase advances between the observation point and the sources at locations $w$. The coefficients $h_{w, j k l m}$ are defined using the normal and skew multipolar strengths $K_{w}$ and $J_{w}$ respectively,

$$
\begin{aligned}
h_{w, j k l m}= & -\frac{\left[K_{w, n-1} \Omega(l+m)+i J_{w, n-1} \Omega(l+m+1)\right]}{j ! k ! l ! m ! 2^{j+k+l+m}} \\
& \times i^{l+m} \beta_{\beta_{x, w}}^{\frac{(j+k)}{(l+m)}} \beta_{y, w}^{\left(\frac{2+m}{2}\right.} \\
& \text { with }\left\{\begin{array}{ll}
\Omega(i)=1 & \text { if } i \text { is even } \\
\Omega(i)=0 & \text { if } i \text { is odd }
\end{array} .\right.
\end{aligned}
$$

The addition of ac dipoles introduces new frequencies that lead to new resonances. The derivations presented in [17] may be extended to two dimensions to obtain the resonance condition for ac dipole driven motion, as

$$
\begin{aligned}
& \left(k_{1}-j_{1}\right) Q_{x}+\left(k_{2}-k_{3}+j_{2}-j_{3}\right) Q_{x}^{a c}+ \\
& \left(m_{1}-l_{1}\right) Q_{y}+\left(m_{2}-m_{3}+l_{2}-l_{3}\right) Q_{y}^{a c}=p \\
& \text { with, } \quad p \in \mathbb{Z} \text { and } j_{i}, k_{i}, m_{i}, l_{i} \in \mathbb{N}_{0},
\end{aligned}
$$

where $Q_{x, y}^{\mathrm{ac}}$ are the horizontal and vertical ac dipole tunes, and the indices fulfill the following conditions, 


$$
\begin{aligned}
j_{1}+j_{2}+j_{3} & =j \\
k_{1}+k_{2}+k_{3} & =k \\
l_{1}+l_{2}+l_{3} & =l \\
m_{1}+m_{2}+m_{3} & =m \\
j_{1}+l_{1} & >0 .
\end{aligned}
$$

In general the number of resonances is larger when exciting the beam with an ac dipole and also include the resonances present in the free motion. The choice of working point for the bare machine tunes as well as for the ac dipole tunes will therefore have a significant influence on the forced dynamic aperture, as explored in Sec. III.

Resonances are approached by the detuning of the natural tunes with amplitude. As the oscillation amplitude increases, the natural tunes will detune on to resonances causing particle losses. Amplitude detuning is in general larger with forced oscillations. It has been shown that the direct linear amplitude detuning terms generated by normal octupolar fields double under the influence of an ac dipole driven motion [15]. The tune shifts for free and driven motion horizontal excitations are given by

$$
\begin{aligned}
\Delta Q_{x} & =\frac{q}{p} \frac{3 B_{4}}{8 \pi}\left(\beta_{x}^{2} J_{x}^{\text {free }}+2 \beta_{x} \beta_{x}^{\prime} J_{x}^{\text {forced }}\right) \\
\Delta Q_{y} & =-\frac{q}{p} \frac{3 B_{4}}{8 \pi}\left(2 \beta_{x} \beta_{y} J_{x}^{\text {free }}+2 \beta_{x}^{\prime} \beta_{y} J_{x}^{\text {forced }}\right),
\end{aligned}
$$

where $q$ and $p$ are the particle charge and momentum, $B_{4}$ is the integrated field of a normal octupole normalized with the magnetic rigidity, $\beta_{x, y}$ are the $\beta$-functions at the octupole sources, $\beta_{x, y}^{\prime}$ are the $\beta$-functions at the octupoles under the influence of the ac dipole motion [7], $J_{x, y}^{\text {free }}$ and $J_{x, y}^{\text {forced }}$ are the actions of the free oscillation and forced oscillation, respectively. When neglecting $\delta_{z,+}$, the forced action can be defined as $J_{x}^{\text {forced }}=\frac{1}{2}\left|\delta_{z,-}\right|^{2}$. The expressions for vertical excitations are obtained by swapping the plane indices. Assuming the optics perturbations coming from the ac dipole are small $\left(\beta_{x, y} \approx \beta_{x, y}^{\prime}\right)$, and that the forced oscillation is much larger than the free oscillation component $\left(J_{x, y}^{\text {forced }} \gg J_{x, y}^{\text {free }}\right)$, Eq. (7) shows that the detuning for forced oscillations is twice as large as for free motion (when $J_{x, y}^{\text {forced }}=0$ ) in the plane of oscillation. Further detuning from higher order nonlinear fields, such as second order detuning from dodecapoles, are even more enhanced [15].

The increased amplitude detuning under forced oscillations combined with the larger number of resonances should result in a forced dynamic aperture typically smaller than the free dynamic aperture $\left(D A_{\text {forced }} \lesssim D A_{\text {free }}\right)$, and can thus give a qualitative lower bound estimate on the free dynamic aperture.

\section{SIMULATIONS OF FORCED DYNAMIC APERTURE WITH AC DIPOLE}

Tracking simulations are done in MAD-X [32] to explore the effect of the ac dipole driving tunes on forced dynamic aperture. For the purpose of this study, the nominal 2016 model of the LHC at top energy $(6500 \mathrm{GeV})$ and end of squeeze $\left(\beta^{*}=0.40 \mathrm{~m}\right)$ is used. Sources of nonlinearities are introduced to the model in the following ways; The nonlinear magnetic errors as generated by Windows Interface to Simulations Errors (WISE) [34] are applied to all dipoles and quadrupoles. The corrector spool pieces settings for the sextupoles (MCS), octupoles (MCO) and decapoles (MCD) are used as implemented during regular 2016 LHC operation. Misalignments of the separation dipoles are introduced, and the orbit is corrected to zero with a residual rms orbit of $2 \times 10^{-6} \mathrm{~m}$. The crossing angles in the interaction regions are turned off and all other parameters such as tunes, chromaticity and coupling are corrected to $Q_{x}=0.31, Q_{y}=0.32, Q_{x, y}^{\prime}=5,\left|C^{-}\right|=$ $5 \times 10^{-4}$, and $\beta^{*}=0.40 \mathrm{~m}$ to represent normal conditions during optics measurements in the LHC. The coupling parameter is defined according to [35] by

$$
C^{-}=\frac{1}{2 \pi R} \int_{0}^{2 \pi} \sqrt{\beta_{x} \beta_{y}} K e^{i\left[\left(\phi_{x}-\phi_{y}\right)-\left(Q_{x}-Q_{y}-p\right) \theta\right]} d \theta
$$

where $R$ is the circumference of the accelerator, $K$ is the skew quadrupolar strength as defined in [35], $\left(Q_{x}-Q_{y}-p\right)$ is the fractional tune difference, $\phi_{x / y}$ are the horizontal and vertical phase advances, $\beta_{x / y}$ are the horizontal and vertical $\beta$-functions, and $\left|C^{-}\right|$is the coupling stopband.

Free kick single particle tracking simulations are done by introducing a $\Delta x$ and/or $\Delta y$ offset as initial conditions and then tracked using the MAD-X thin lens tracking module. For the ac dipole single particle tracking simulations the particle is initially at rest $\left(x=p_{x}=y=p_{y}=0\right)$. The ac dipole excitation starts after 500 turns and the excitation amplitude is ramped up for 2000 turns after which it is kept constant for 6000 turns (flattop) and finally ramped down for 2000 turns till zero amplitude. Figure 1 shows the turnby-turn signal for the particle at BPM.22L1.B1 for the vertical plane as used in simulations. Those parameters are comparable to what is currently used for the ac dipole in the LHC.

Dynamic aperture from simulations is commonly represented as the stable domain as a function of actions, where the actions are obtained from the initial conditions. Such a representation may not be trivially obtained for ac dipole excitations where the particle is initially at rest.

Two methods are used in the paper to measure the actions from turn-by-turn data in both simulations and measurements. In the case of single particle tracking simulations, multiparticle simulations with ac dipole, and measurements with ac dipole, where a sustained coherent oscillation is 


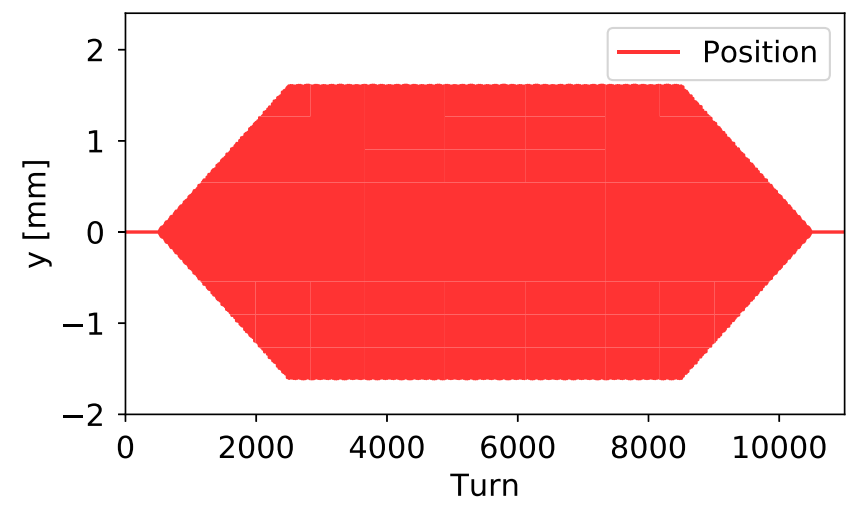

FIG. 1. Typical ac dipole excitation showing the turn-by-turn amplitude of a particle in the vertical plane. The excitation is similar in the horizontal plane.

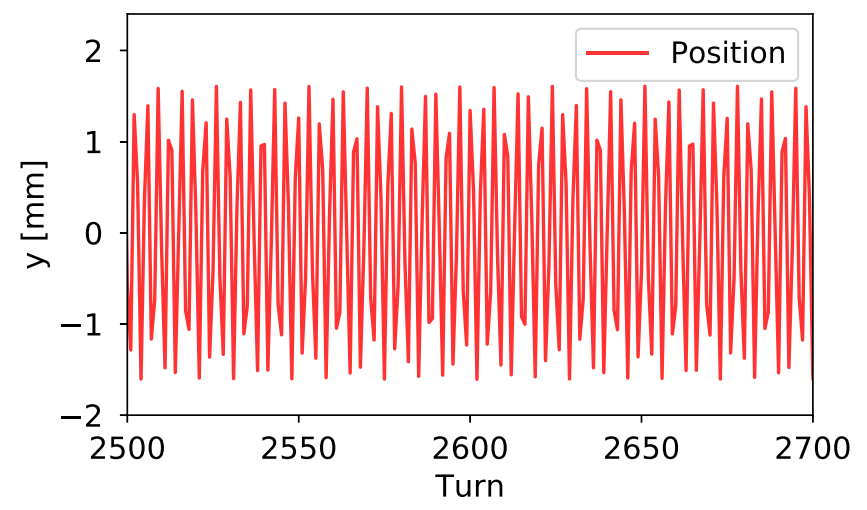

FIG. 2. A close-up plot of the first 200 turns of the flattop excitation showing the coherent turn-by-turn oscillation with an amplitude of $A=1.6 \mathrm{~mm}$.

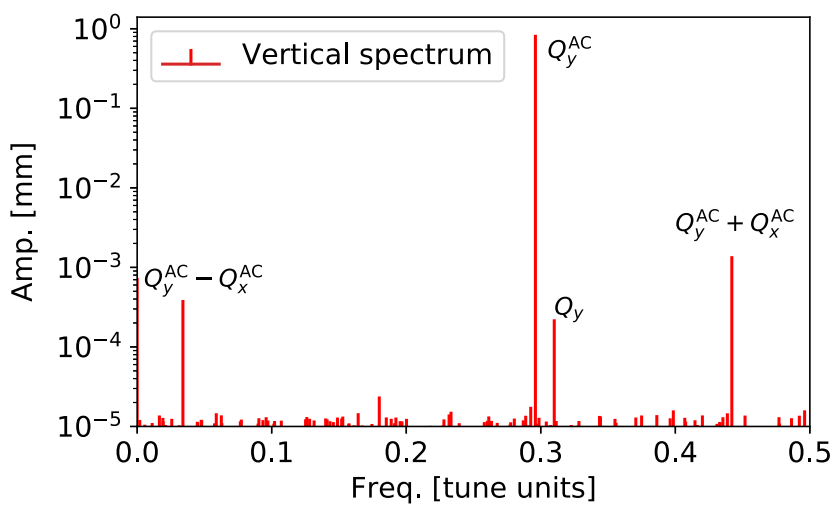

FIG. 3. Spectrum of the flattop turn-by-turn data. The main spectral line is measured at the ac dipole vertical tune $Q_{y}^{\mathrm{ac}}=$ 0.296 and with an amplitude of $A^{\prime}=0.80 \mathrm{~mm}$, i.e. $A=2 A^{\prime}$.

observed in the turn-by-turn data, the actions are measured from the amplitude of the tune line in the spectrum of the BPM turn-by-turn tracking data. The spectral analysis code SUSSIX [36] is used for all spectral analysis in this paper.
However for free kick measurements where large sources of nonlinearity cause the beam to decohere quickly, the usable number of turns for the spectral analysis becomes too small. The quality of spectral analysis quickly deteriorates, and the actions are thus determined from the peak-to-peak amplitude of the turn-by-turn signal.

In single particle simulations, where both free and forced oscillations yield coherent turn-by-turn position data, the actions are calculated by spectral analysis of the turn-byturn data of the first 200 turns for the free kick motion, and the first 200 turns of the flattop data of the ac dipole excitations. Only the first 200 turns are used to mitigate the effects of possible emittance growth when exciting close to resonances. Figure 2 shows the first 200 turns of flattop turn-by-turn data for a vertical excitation with an oscillation amplitude of $A=1.6 \mathrm{~mm}$. The spectrum of the this turn-by-turn data is shown in Fig. 3. The amplitude signal $A^{\prime}$ of the main tune line at frequency $Q_{y}^{\text {ac }}$ is observed with an amplitude of $A^{\prime}=0.80 \mathrm{~mm}$ in the spectrum and is proportional to the signal amplitude with $A=2 A^{\prime}$. The actions are calculated with

$$
2 J_{x, y}=\frac{A^{2}}{\beta_{x, y}}
$$

The actions are then normalized to the emittance using

$$
N=\sqrt{\frac{2 J_{x, y}}{\epsilon_{x, y}}},
$$

where $\epsilon_{x, y}$ are the physical emittances and are defined as $\epsilon_{x, y}=7.8 \times 10^{-9} \mathrm{~m}$ at injection energy $(0.45 \mathrm{TeV})$, and $\epsilon_{x, y}=5.4 \times 10^{-10} \mathrm{~m}$ at top energy $(6.5 \mathrm{TeV})$.

Figure 4 shows the free dynamic aperture as a function of initial conditions, while the free dynamic aperture as a function of the calculated actions from the main spectral line is shown in Fig. 5. Both results are from the same free kick simulations with 6000 turns. The blue points represent surviving particles and the red points are particles lost during tracking. Certain regions in action space are not fully probed using the usual regular phase space sampling. At large excitation amplitudes, nonlinearities start to distort the sampling from initial conditions and does not guarantee a smooth coverage in action space.

Simulations allow to probe a large area of phase space and define the forced and free dynamic apertures as the minimum radial distance to the first observed losses. The free dynamic aperture is simulated at $12.2 \sigma_{\text {nom }}$ as shown by the black and white arc in Fig. 5. This is slightly lower than the $13.8 \sigma_{\text {nom }}$ free dynamic aperture found in Fig. 4 from the initial conditions. 


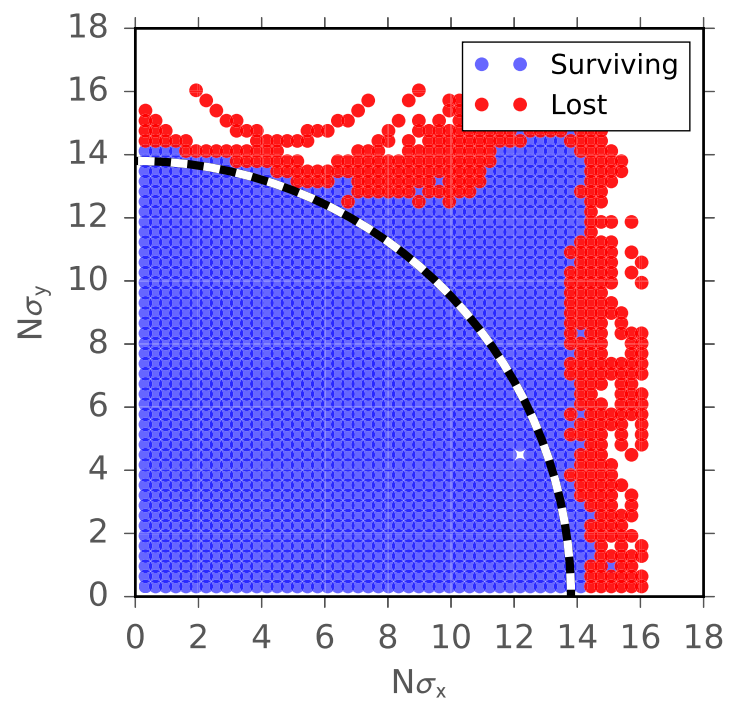

FIG. 4. Simulated free dynamic aperture with excitations calculated from initialized amplitude. The machine tunes are $Q_{x}=0.31$ and $Q_{x}=0.32$.

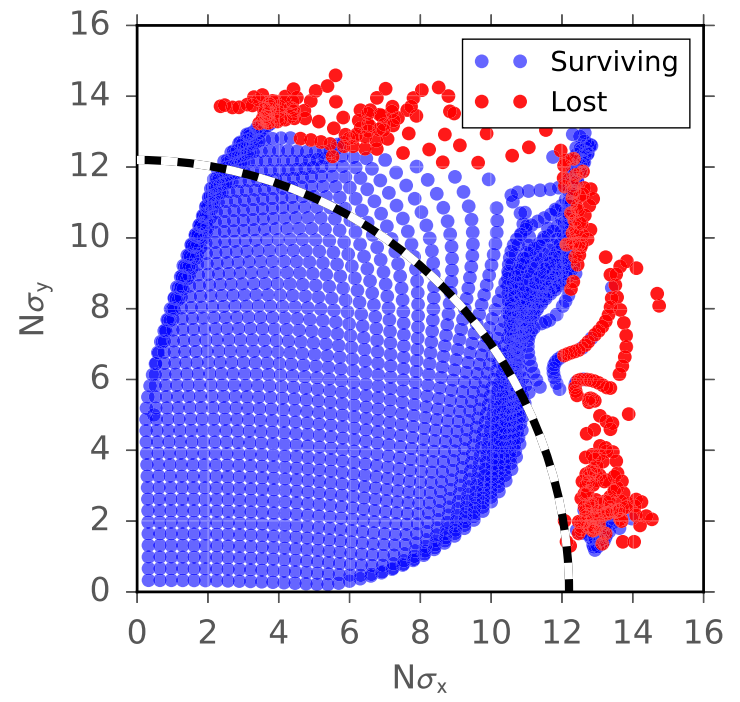

FIG. 5. Simulated free dynamic aperture with excitations calculated from the amplitude of the main spectral line. The machine tunes are $Q_{x}=0.31$ and $Q_{x}=0.32$.

\section{A. Effect of driving tunes on forced dynamic aperture}

Figure 6 shows the simulated forced dynamic aperture for different ac dipole driving tunes. The top figure shows the simulated forced dynamic aperture for symmetric excitation of the ac dipole where $Q_{x}^{\mathrm{ac}}=Q_{x}-0.012$ and $Q_{y}^{\mathrm{ac}}=Q_{y}+0.012$. The forced dynamic aperture is limited to $6.2 \sigma_{\text {nom }}$ due to early losses for diagonal excitations as the octupolar resonance $Q_{x}^{\mathrm{ac}}+Q_{y}^{\mathrm{ac}}-Q_{x}-Q_{y}=p$, with $p$ as integer, is excited. This resonance is automatically excited at low amplitudes, though at larger amplitudes its excitation
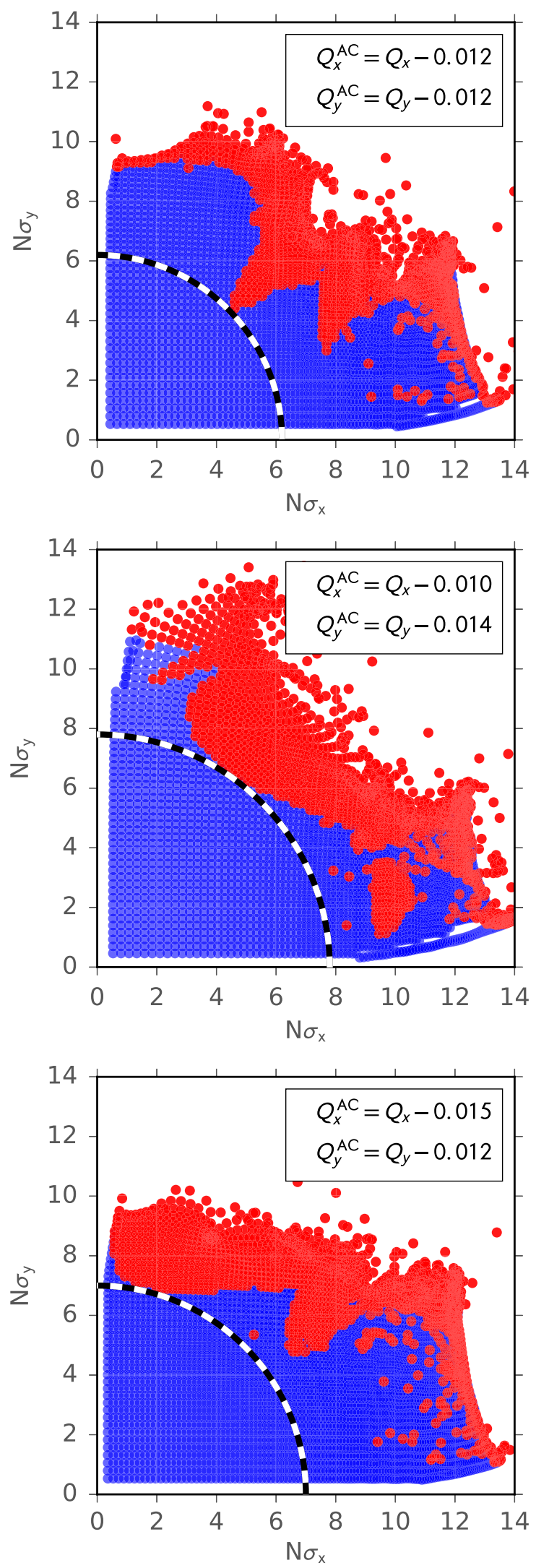

FIG. 6. Forced dynamic aperture results for three different ac dipole driving tunes at top energy the natural tunes are $Q_{x}=0.31$ and $Q_{y}=0.32$. 

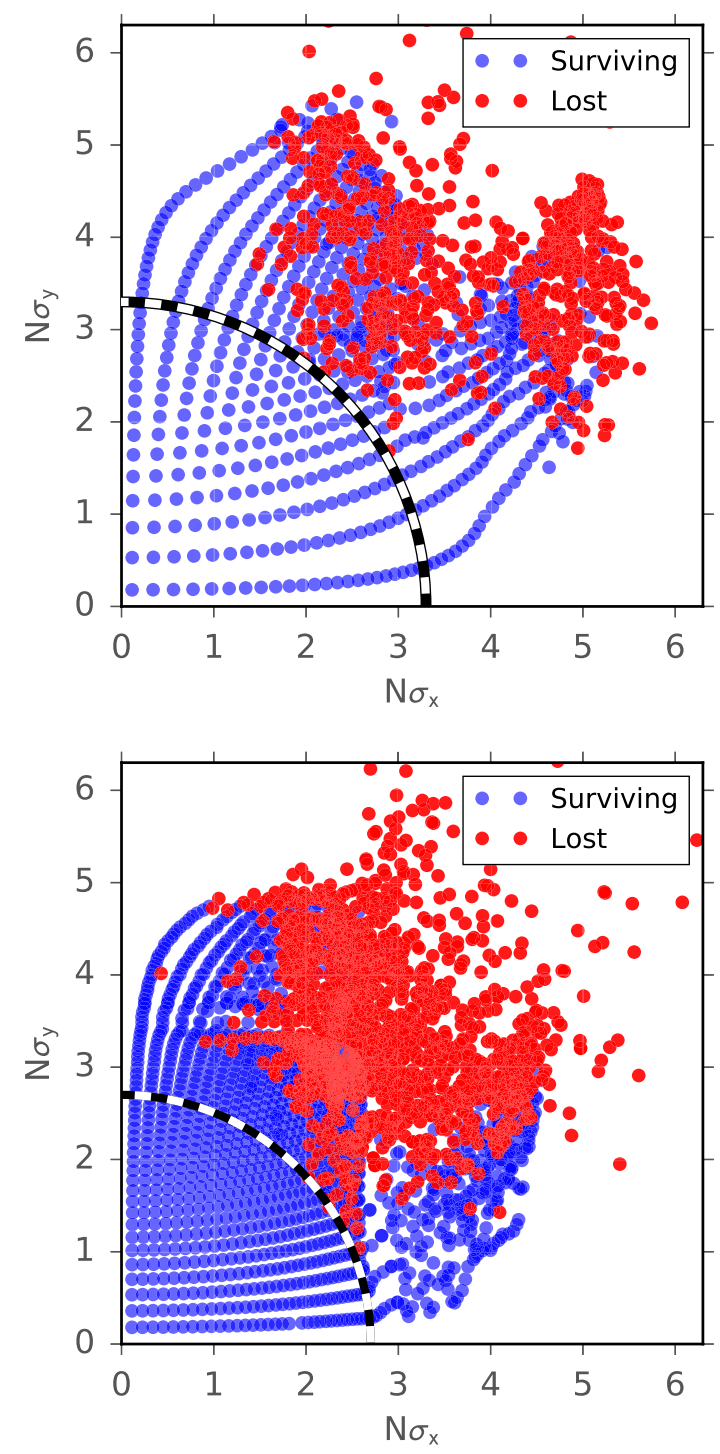

FIG. 7. Forced dynamic aperture at injection with Landau octupoles at $40 \mathrm{~A}$. The top figure shows the forced dynamic aperture for a model including magnetic errors, while the bottom figure shows the forced dynamic aperture for a model including geometrical rotational errors as well as magnetic errors. A reduction of forced dynamic aperture is observed when including the geometrical rotational errors in the multipoles.

depends on symmetric amplitude detuning in both planes. Results of tracking simulations with two different working points are also presented in Fig. 6 with $Q_{x}^{\text {ac }}=Q_{x}-0.010$ and $Q_{y}^{\mathrm{ac}}=Q_{y}+0.014$ (middle), and $Q_{x}^{\mathrm{ac}}=Q_{x}-0.015$ and $Q_{y}^{\mathrm{ac}}=Q_{y}+0.012$ (bottom), where their respective forced dynamic aperture are found at $7.8 \sigma_{\text {nom }}$ and $7.0 \sigma_{\text {nom }}$. In all three cases the forced dynamic aperture is lower than the free dynamic aperture, confirming qualitative predictions from Sec. II. All three simulations with different ac dipole driving tunes show a considerably different forced dynamic aperture. It is clear from these results that the choice of ac dipole driving tunes has a large effect on the forced dynamic aperture and that the angle at which first losses occur is varying. These differences show that to accurately use the forced dynamic aperture as an observable for nonlinearities the same working point should be used throughout all measurements to compare forced dynamic aperture at different nonlinear configurations. Furthermore, it should be emphasized that the choice of working point can greatly increase the available aperture for optics measurements with ac dipole, a fact that will be relevant for the High Luminosity LHC where nonlinear errors are expected to be large.

\section{B. Simulations at injection energy}

Single particle tracking simulations of forced dynamic aperture at injection energy $(450 \mathrm{GeV})$ with operational Landau octupole strengths of 2016 [37] are shown in Fig. 7 for two different models. The top figure shows the forced dynamic aperture for the nominal injection model with magnetic errors applied in the same way as detailed previously, and the Landau octupoles powered to $40 \mathrm{~A}$. The minimum forced dynamic aperture is simulated at $3.3 \sigma_{\text {nom }}$. A large distortion of the probed action space is observed at large amplitudes due to the very strong Landau octupoles at injection energy. The bottom figure shows the forced dynamic aperture for simulations with the same model and with the addition of rotational geometric errors in all multipoles. After the introduction of the geometrical rotational errors the coupling is corrected to $\left|C^{-}\right|=$ $5 \times 10^{-4}$, a typical value during optics measurements in the LHC. A reduction of forced dynamic aperture to $2.6 \sigma_{\text {nom }}$ is observed. Geometrical rotations of multipoles change the nonlinear content of the model, which translates to a change in simulated forced dynamic aperture.

\section{EVALUATION OF FORCED DYNAMIC APERTURE FROM BEAM INTENSITY LOSSES}

Both free and forced short term dynamic aperture can be measured by probing the beam intensity loss after large transverse excitations. In general, as the excitation amplitude is increased, more particles will cross the free or forced dynamic aperture and become lost. By assuming that the losses are dominant in a single plane and that the free or forced dynamic aperture is smooth, the problem can be simplified to a single dimension. In such a case the condition for loosing a particle from free DA becomes,

$$
J^{\text {free }}>D A_{\text {free }}
$$

and for loosing a particle from forced DA

$$
J^{\text {free }}+J^{\text {forced }}>D A_{\text {forced }}
$$

This is reflected in Figs. 8 and 9. Figure 8 shows a beam excited with a single free kick. All particles beyond the free dynamic aperture are lost. The free dynamic aperture is 


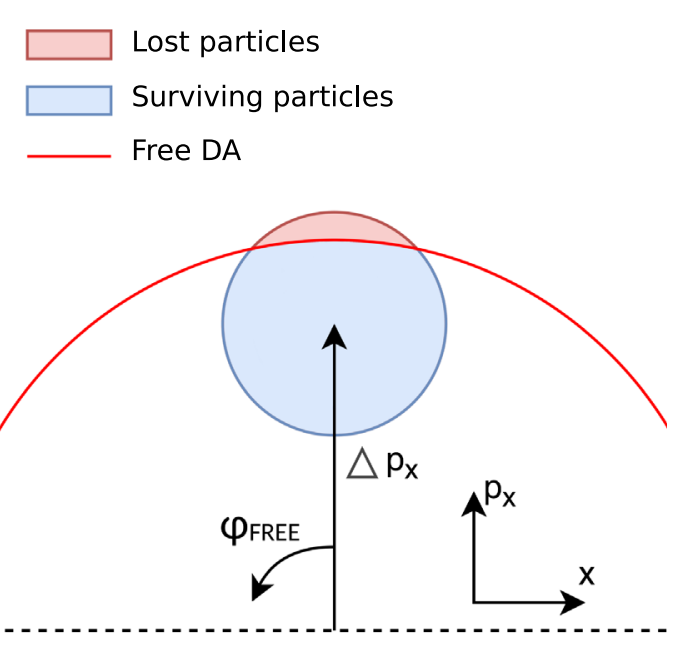

$(0,0)$

FIG. 8. Diagram showing where the losses in the particle bunch occur as a result of reaching the free dynamic aperture. As the kick amplitude $\left(\Delta p_{x}\right)$ increases more particles will cross the free dynamic aperture and will be lost.

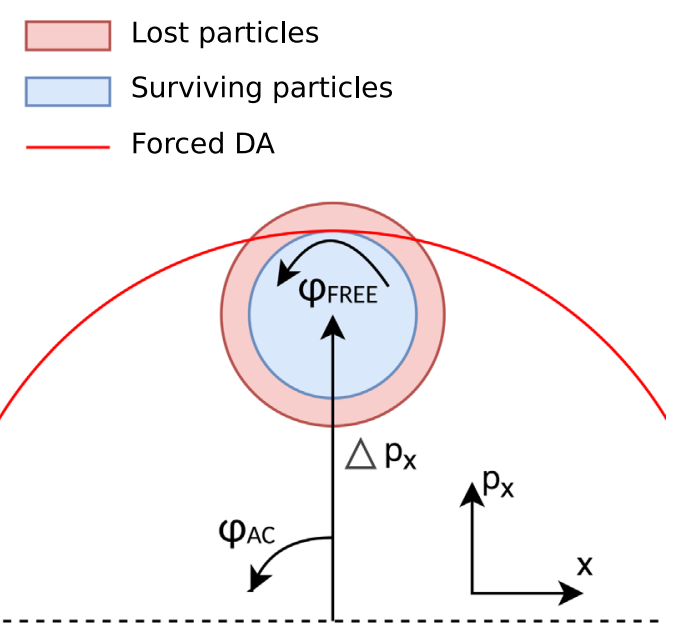

$(0,0)$

FIG. 9. Diagram showing where the losses in the particle bunch occur as a result of reaching the forced dynamic aperture. The remaining free motion inside the bunch will cause the tails of the bunch to be lost.

determined by fitting an error function to the measured losses over kick amplitudes as described in [38-40].

The coherent excitation of the ac dipole will not change the initial bunch profile. Multiparticle tracking simulations are done to verify that the Gaussian bunch profile is maintained during the ac dipole excitation. Figure 10 shows the Gaussian bunch profile with 4000 particles for a bunch at rest before the ac dipole excitation and the same particles during the flattop excitation of the ac dipole. The bunch size is calculated before the ac dipole excitation at $\sigma=1.305 \mathrm{~mm}$, and during the flattop ac dipole excitation at $\sigma=1.309 \mathrm{~mm}$. The small deviations between the two

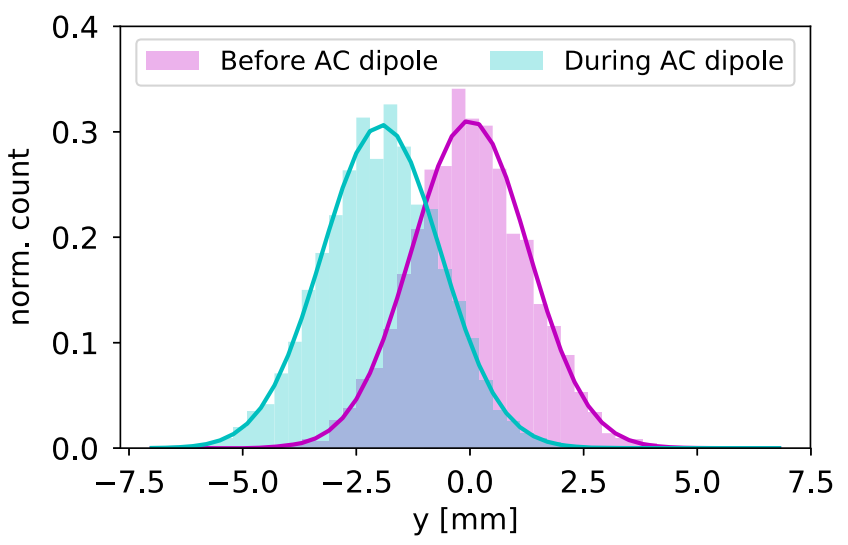

FIG. 10. Bunch distributions for vertical excitations of ac dipole for the case before the ac dipole (at rest) and during the flattop ac dipole excitation for multiparticle tracking simulations with 4000 particles. Before the ac dipole the bunch size is $\sigma=1.305 \mathrm{~mm}$, and during the flattop ac dipole excitation it is measured at $\sigma=1.309 \mathrm{~mm}$.

are attributed to limited number of particles used in the simulation. This is also representative for the other coordinates $x, p_{x}$, and $p_{y}$.

Figure 9 shows an ac dipole excited beam traversing the forced dynamic aperture. In contrast to the free kick case where only particles beyond the free dynamic aperture are lost, the particle motion with ac dipole excitation will cause all the tails to be lost. By further assuming that the coupling is negligible this simplifies the problem to an integral over the distribution in action space only,

$$
\frac{\Delta I}{I}\left(D A_{\text {forced }}\right)=\int_{D A_{\text {forced }}}^{+\infty} \frac{1}{\epsilon_{z}} e^{-\frac{w-J_{\text {forced }}}{\epsilon_{z}}} d w
$$

where $\Delta I / I$ is the normalized measured losses, $J_{z}^{\text {forced }}$ is the measured action of the bunch from forced oscillations, $\epsilon_{z}$ is the beam emittance, $w$ is the integration variable in units of $J_{z}^{\text {forced }}$, and $z \in\{x, y\}$ determines the plane of losses. This leads to the following expression of the forced dynamic aperture for given beam losses and forced excitation

$$
D A_{\text {forced }}=J_{z}^{\text {forced }}-\epsilon_{z} \ln \left(\frac{\Delta I}{I}\right) .
$$

which is dependent on $J_{z}^{\text {forced }}$ and $\Delta I / I$. The resulting expression for the forced dynamic aperture is very close to the exponential formula for free dynamic aperture as defined in [30], but now contains a kick term ( $\left.J_{z}^{\text {forced}}\right)$. The forced dynamic aperture can then be calculated from measurements by fitting Eq. (14) to the measured beam intensity losses over ac dipole excitation amplitudes.

Further simulations are done with an evenly sampled vertical phase space and the same conditions as presented in Sec. III B. The horizontal initial conditions are set to 


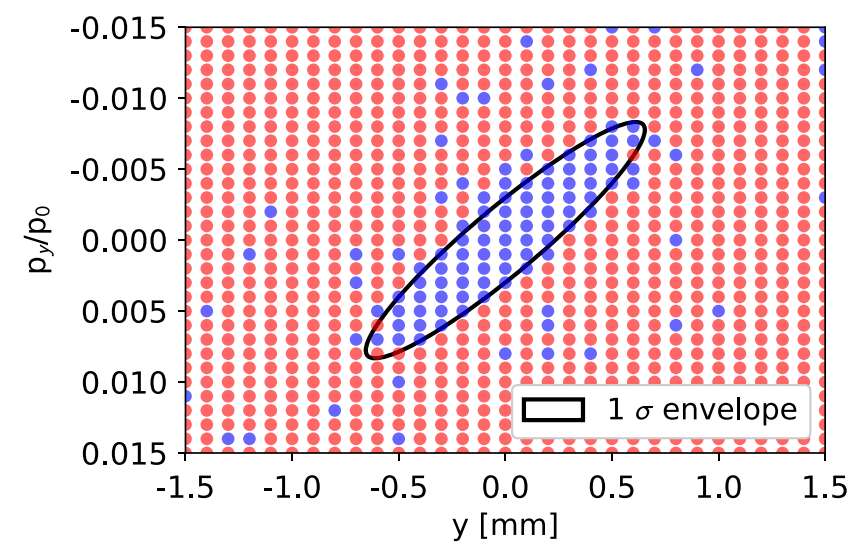

FIG. 11. Location of losses from forced DA with evenly sampled vertical phase space. In black the phase space ellipse at $1 \sigma$ is drawn for reference.

$x=p_{x}=0$. All particles are excited with the same diagonal excitation close to the forced dynamic aperture. Figure 11 shows the lost and surviving particles as a function of their initial conditions in the vertical plane. All tails of the initial distribution are lost due to the forced dynamic aperture which is in line with the representation of Fig. 9.

\section{FORCED DYNAMIC APERTURE MEASUREMENTS AT INJECTION ENERGY}

A first demonstration of forced dynamic aperture measurements using the LHC ac dipoles at injection energy is presented. The experiments carried out consist of exciting the beam with the LHC aperture kicker (MKA) or the LHC ac dipoles and measuring the beam intensity losses using the LHC beam current transformer system (BCT) [41]. A single pilot bunch is used during the measurements and the collimators are retracted to $12 \sigma_{\text {nom }}$ to allow for large excitations without scraping the stable beam and providing the necessary aperture to probe the free and forced dynamic aperture. Measurements were performed with the Landau octupoles powered at $40 \mathrm{~A}$, equal to the operational settings in 2016 [37] as well as at 6.5 A. Several series of measurements were done in Beam 2 by exciting the beam in the horizontal plane and vertical planes separately. For the series of free kick measurements a dump and re-inject scheme is used, while during the ac dipole excitations the bunch is dumped and re-injected whenever significant losses occur. The emittance of the injected beams shows no significant variations between different injections and was measured consistently around $1.2 \mu \mathrm{m}$ with the wire scanners in both planes. Free dynamic aperture measurements were first performed with the aperture kicker (MKA) [9] to obtain a free dynamic aperture reference with a conventional free kick method. The experiment was repeated in both planes using the ac dipole.

ac dipole excitation amplitudes and frequencies are controlled through an online measurement tool. The ac

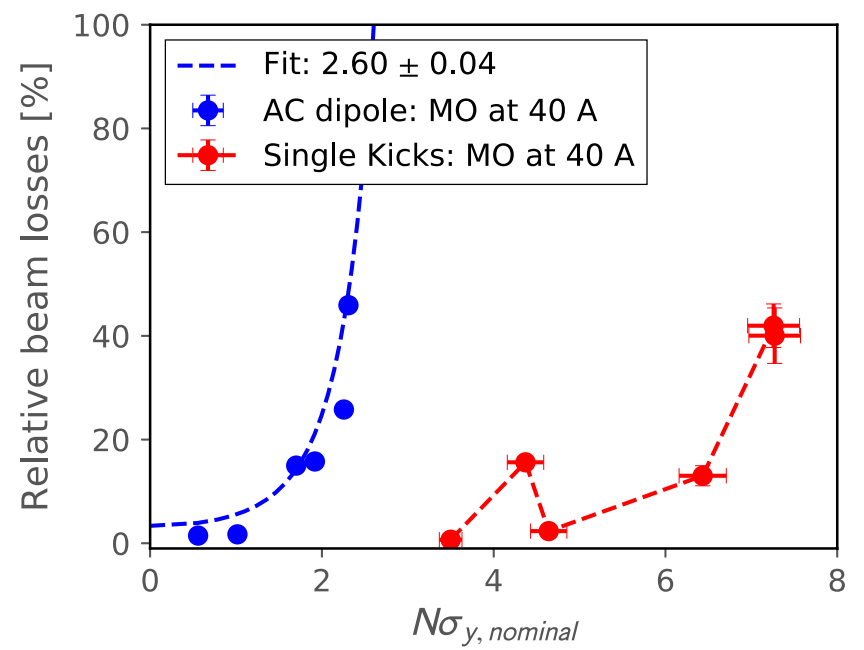

FIG. 12. Measured losses for ac dipole excitations (blue) and free kick (red) measurements with Landau octupoles powered at $40 \mathrm{~A}$.

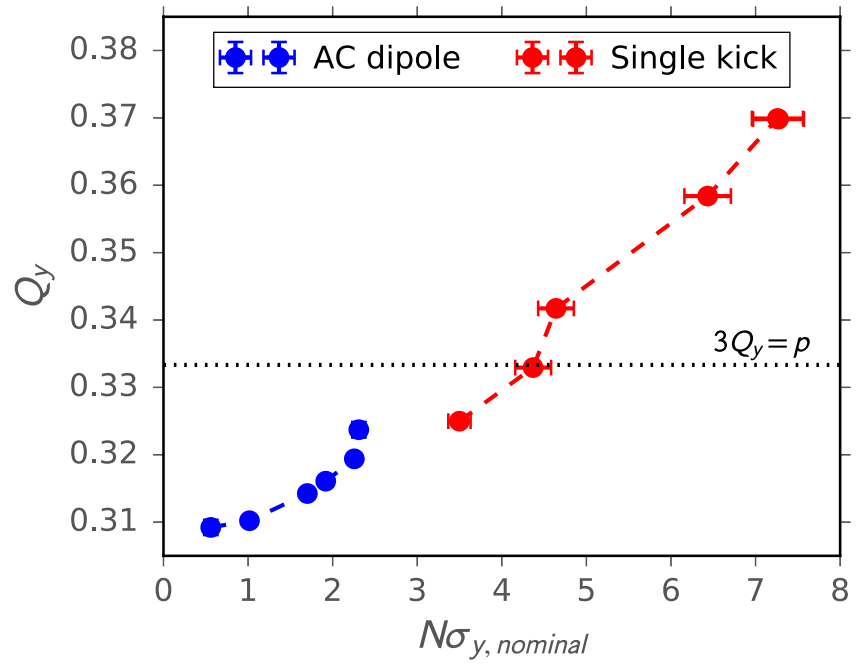

FIG. 13. Measured natural vertical tunes vs vertical excitation amplitudes for measurements with ac dipole (blue) and free kicks (red) and Landau octupoles powered at $40 \mathrm{~A}$.

dipole currents have been demonstrated to be very well regulated with a noise floor at $5 \times 10^{-4}$ of the main frequency amplitude and an emittance growth below the $0.1 \%$ level for the excitation ranges used in the LHC [10]. The ac dipole driving tunes was set to $Q_{y}^{a c}=Q_{y}-0.014=$ 0.296 and $Q_{x}^{a c}=Q_{x}-0.018=0.262$ to ensure the $Q_{y}^{a c}=Q_{y}$ resonance is not approached through detuning with amplitude for vertical excitations. The measurement was repeated with lower octupole strength $(+6.5 \mathrm{~A})$ to probe the change of forced dynamic aperture.

Figure 12 shows the measured beam losses as percentages of initial beam intensity for measurements with Landau octupoles at 40 A with free kick and ac dipole excitations in the vertical plane in the top plot and the 
corresponding measured natural tunes in the bottom plot. The actions are calculated at each BPM along the ring by using the main spectral line amplitude for ac dipole excitations and the peak-to-peak of the turn-by-turn signal for the free kicks as discussed in Sec. III. In practice, an average over all arc BPMs is taken, having the benefit of providing an error bar for the action measurement and excluding the BPMs in the insertion regions. The measurement errors on the actions are defined as the standard deviation of the measured actions in all working arc BPMs. The measured natural tunes are measured from the turn-byturn data at each BPM using the spectral analysis code SUSSIX. The error is defined as the standard deviation over all BPMs and is in general small.

The beam losses are calculated from the BCT measured intensity before and after excitation. In the case of the ac dipole excitation this gives very clear losses from the BCT data, as there are no beam losses after excitation. For free kicks the difference in beam intensity is measured at the same timescales as the ac dipole excitation. However, continued slow losses are occurring then due to the free dynamic aperture. This results in a larger measured error on the relative beam losses for free kicks.

The forced dynamic aperture is calculated by fitting Eq. (14) to the measured losses as a function of ac dipole excitation amplitudes. The fit is also shown in Fig. 12 as the blue dotted line. We observe a very good agreement between measurement and the fit. The forced dynamic aperture is measured with the ac dipole at $(2.60 \pm 0.04) \sigma_{\text {nom }}$ and is limited by the 3 rd order resonance $3 Q_{y}=p$, as shown in Fig. 13. In the case of free kicks, the free dynamic aperture arising from the $3 \mathrm{rd}$ order resonance is $4.3 \sigma_{\text {nom }}$. The kicks at 3.5 and $4.6 \sigma_{\text {nom }}$ show very small losses thus enclosing a thin unstable region at the $3 \mathrm{rd}$ order resonance. Such a thin unstable region explains the small losses observed for free kicks at the free dynamic aperture. Further free kick measurements at larger amplitude were performed up to $7.3 \sigma_{\text {nom }}$ where the beam is lost. As free kicks apply an instant change in phase space, it is possible to excite the beam beyond the 3rd order resonance without crossing the resonance for large amplitude kicks. It should be noted that such strong kicks are not present in operational conditions and that any bunch experiencing a gradual increase of amplitude will thus cross the resonance and become resonantly excited. In the case of the ac dipole the 3rd order resonance is dominant in defining the forced dynamic aperture. Due to the ramp up of the ac dipole all particles will slowly cross the resonance and thus be lost.

Figure 14 shows the beam losses for vertical kicks with ac dipoles at two different settings of Landau octupoles. The first curve, in blue, shows the same results as in Fig. 12 for Landau octupoles powered at $40 \mathrm{~A}$. The forced dynamic aperture for measurements with lower octupole powering $(6.5 \mathrm{~A})$ is shown in green, and forced dynamic aperture from the fit is calculated at $(6.86 \pm 0.12) \sigma_{\text {nom }}$.

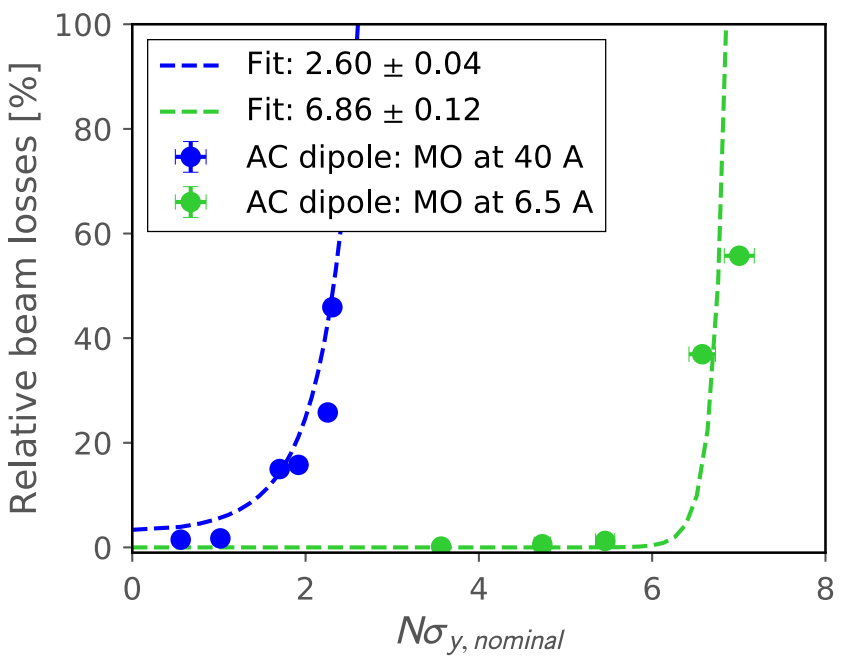

FIG. 14. Measured losses for ac dipole excitations with Landau octupoles powered at $40 \mathrm{~A}$ (blue) and $6.5 \mathrm{~A}$ (green).

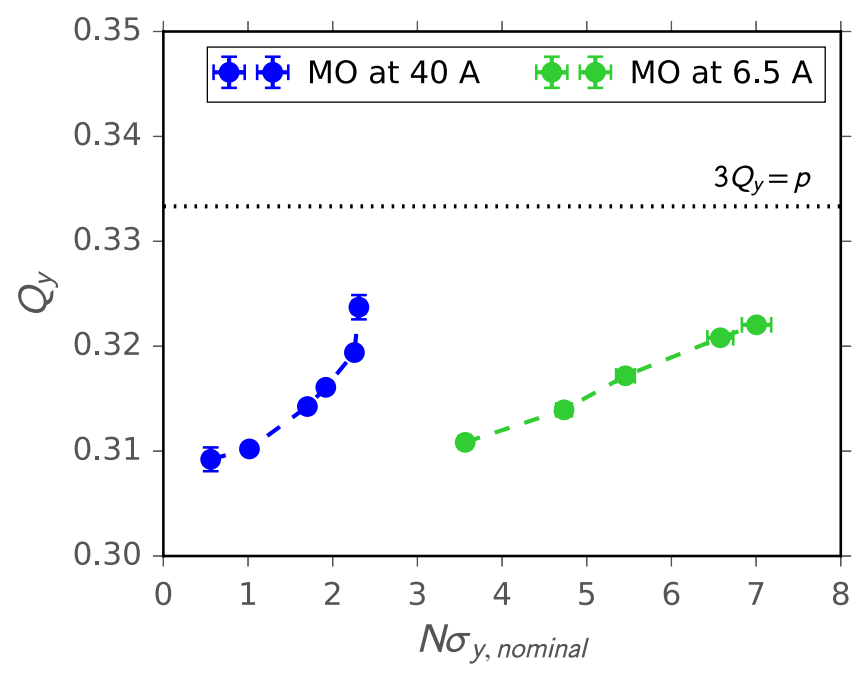

FIG. 15. Measured natural vertical tunes vs vertical excitation amplitudes for measurements with ac dipole and Landau octupoles powered at $40 \mathrm{~A}$ (blue) and $6.5 \mathrm{~A}$ (green).

An increase of forced dynamic aperture is observed with a reduction of Landau octupole strength. This can be related to much weaker amplitude detuning coming from the Landau octupoles, as shown in Fig. 15. Amplitude detuning is linear in octupole current and quadratic in $\sigma_{\text {nom }}$. The expected increase of forced dynamic aperture from reduced amplitude detuning with weaker octupoles is thus $\sqrt{6.5 / 40}=2.48$, while the observed increase of $D A_{\text {forced }}(40) / D A_{\text {forced }}(6.5)=2.64$. In both cases the forced dynamic aperture is limited by the 3 rd order resonance. The measurements demonstrate that changes in the nonlinear state of the machine can be measured with forced dynamic aperture measurements. 


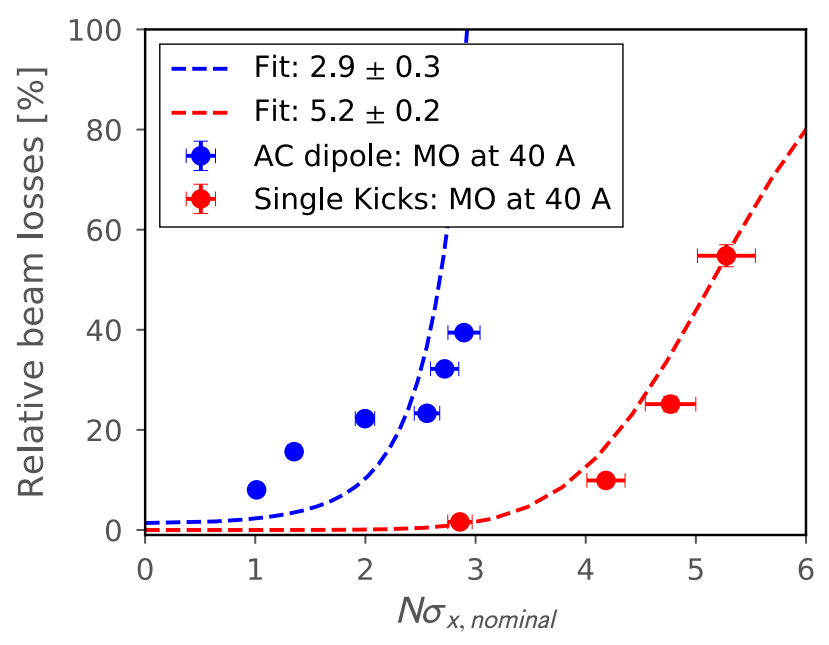

FIG. 16. Measured losses for both free kicks (red) and ac dipole excitations (blue) in the horizontal plane with Landau octupoles powered at $40 \mathrm{~A}$. The blue fit is done with Eq. (14), while the red fit is done with the error-function of [40].

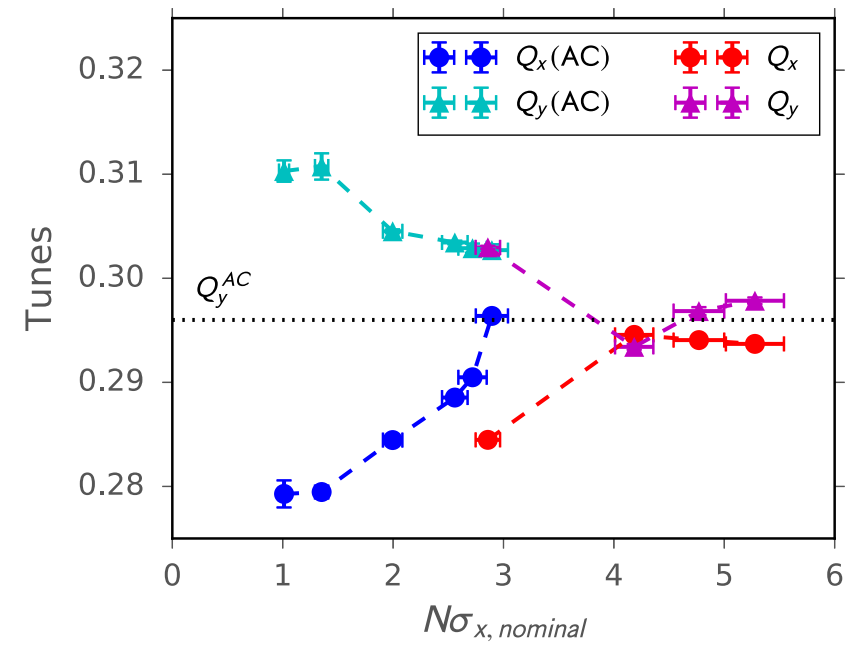

FIG. 17. Measured natural horizontal and vertical tunes vs horizontal excitation amplitudes with Landau octupoles powered at $40 \mathrm{~A}$ for ac dipole excitations and free kicks excitations.

Further measurements with horizontal excitations are presented in Fig. 16. The resulting particle losses for measurements at horizontal excitations with both the ac dipole (in blue) and the MKA (in red) are shown. The forced dynamic aperture in the horizontal plane for the ac dipole was measured at $(2.9 \pm 0.3) \sigma_{\text {nom }}$, while the free dynamic aperture was measured at $(5.2, \pm 0.2) \sigma_{\text {nom }}$. Note that the fit for the free dynamic aperture is done with the error function as described in [40]. In the case of the ac dipole driven motion, the horizontal natural tune is detuning onto the linear resonance $Q_{x}=Q_{y}^{a c}$ and nonlinear resonance $2 Q_{x}=2 Q_{y}^{a c}$, as shown in Fig. 17. Most likely, both linear and nonlinear resonances influence the observed particle losses. The free dynamic aperture is reached when approaching the difference linear coupling resonance $Q_{x}=Q_{y}$ and octupolar resonance $2 Q_{x}=2 Q_{y}$ as both the vertical and horizontal tunes approach each other, as presented in Fig. 17. The losses are most likely caused by the octupolar resonance, as the difference linear coupling resonance is a stable resonance.

\section{A. Comparison to multiparticle tracking simulations}

The nonlinear content of the machine can be probed by comparing measurements to simulation. By simulating the beam intensity losses after ac dipole excitation in multiparticle simulations a direct comparison to measurements can be made. Multiparticle bunches are initialized as a Gaussian distribution with 10000 particles following [42] and tracked in MADX. The intensity losses and turn-byturn data of the centroid of charge are observed just like in measurements. The actions are calculated in the same way as with the measurements, by using the turn-by-turn data of the centroid of charge. Multiparticle tracking simulations are done at injection energy with Landau octupole at $40 \mathrm{~A}$ with the two models described in Sec. III B. Figure 18 shows the comparison between measurement and multiparticle simulations for vertical ac dipole excitations. The simulations with the nominal injection model using only magnetic errors are shown in pink. The nonlinear model used in this case fails to reproduce the observed losses in the forced dynamic aperture measurement. The sources of this discrepancy can be related to the fact that the skew sextupolar sources present in the model are much smaller than the beam based observations. The forced dynamic aperture using a model with geometrical rotational errors is shown in light blue. The second model is in good agreement with the measurements. This exercise demonstrates that the nonlinear content of the machine may be probed by directly comparing to simulation, and that improvements in the machine models may be derived from forced dynamic aperture measurements. As this method is allowed at top energy in the LHC this provides a distinct advantage over the conventional single kick methods that are currently forbidden at top energy in the LHC.

Simulations for the case with lower Landau octupole strengths show a similar behavior as with the large octupole powering, as presented in Fig. 19. When the model without rotational lattice errors is used forced dynamic aperture is at $10.8 \sigma_{\text {nom }}$, and does not reproduce the measurements. After including the rotational lattice errors the forced dynamic aperture reduces to $8 \sigma_{\text {nom }}$ and is limited by the third-order resonance $3 Q_{y}$. Though this is still $1 \sigma$ away from the measured values it shows a significant improvement in the model. Excitations at larger amplitudes show reduced losses at around $35 \%$ until the bunches are fully lost again. The losses observed between $8 \sigma_{\text {nom }}$ and $10.8 \sigma_{\text {nom }}$ occur 


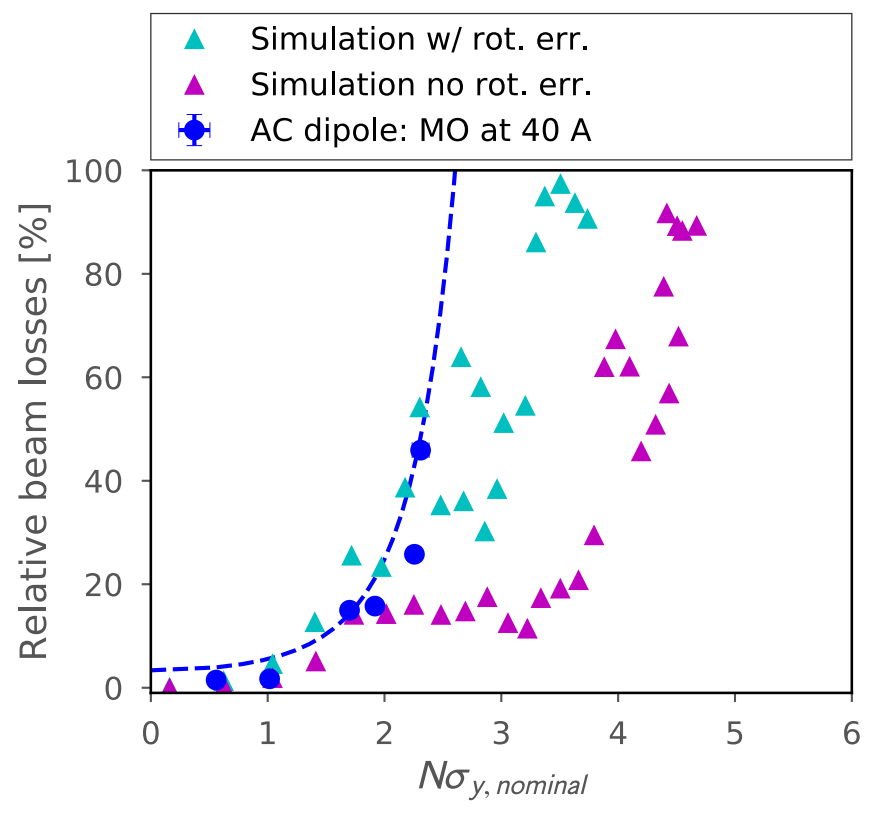

FIG. 18. Measured losses for ac dipole excitations with Landau octupoles at 40 A from measurements and multiparticle simulations. The simulations results without geometrical rotational lattice errors are shown in pink, while the simulations including those rotational errors are shown in light blue.

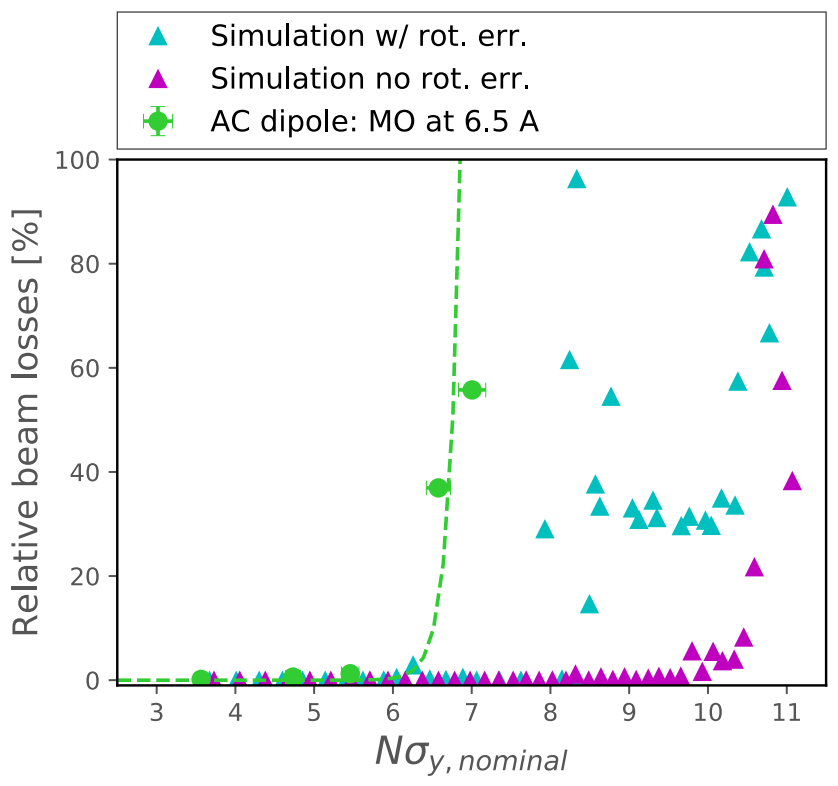

FIG. 19. Measured losses for ac dipole excitations with Landau octupoles at $6.5 \mathrm{~A}$ from measurements and multiparticle simulations. The simulations results without geometrical rotational lattice errors are shown in pink, while the simulations including those rotational errors are shown in light blue.

during the ramp-up and ramp-down of the ac dipole during which the $3 Q_{y}$ resonance is crossed. Future studies will be needed for a more accurate model and exploration of advanced features of this method.

\section{CONCLUSIONS}

In recent years ac dipoles have obtained a prominent role in the commissioning of the LHC, and have been used or envisaged for many other machines. The forced dynamic aperture may become a limiting factor for future applications of ac dipoles. Fortunately the forced dynamic aperture can also be used as a new alternative observable for probing machine nonlinearities.

The nonlinear dynamics under the influence of an ac dipole are altered and the measured forced dynamic aperture is expected to be smaller than the free dynamic aperture. This is related to the appearance of new resonances and to the doubling of first order direct detuning terms in the ac dipole driven motion. Assuming direct detuning terms are the dominant detuning terms, this means enclosing resonances are approached with actions twice smaller than under free oscillations, thus narrowing the stability region in phase-space.

Single particle tracking simulations were done for both forced and free dynamic aperture. At top energy and without Landau octupoles the free dynamic aperture is $12.2 \sigma_{\text {nom }}$. The forced dynamic aperture in single particle tracking simulations with ac dipole is found to be between 6.2 and $7.8 \sigma_{\text {nom }}$ depending on the chosen working point. Results show that the measured forced dynamic aperture is significantly reduced compared to the free dynamic aperture, confirming expectations. Furthermore, the role of the choice of ac dipole driving tunes is demonstrated. Depending on the working point, various resonances are observed to reduce the minimum forced dynamic aperture. It is thus crucial to use the same working point to probe and compare the forced dynamic aperture of different machine configurations. Secondly, this motivates a study to find a working point that maximizes forced dynamic aperture to facilitate optics measurements in the LHC and High Luminosity LHC.

A first demonstration of forced dynamic aperture measurements is presented at injection energy with Landau octupoles powered to $40 \mathrm{~A}$, and later to $6.5 \mathrm{~A}$. At $40 \mathrm{~A}$, measurements with the aperture kicker (MKA) showed a vertical kick free dynamic aperture at the 3rd order resonance at $4.3 \sigma_{\mathrm{nom}}$. The ac dipole forced dynamic aperture was measured at $(2.60 \pm 0.04) \sigma_{\text {nom }}$, limited also by the 3rd order resonance. Lowering of the Landau octupole powering to $6.5 \mathrm{~A}$ resulted in a significant measurable increase of forced dynamic aperture. A reduction of octupole currents to $6.5 \mathrm{~A}$ shows an increase of forced dynamic aperture to $(6.86 \pm 0.12) \sigma_{\text {nom }}$, and illustrates the potential use to characterize relative changes in the nonlinear state of the machine using forced dynamic aperture measurements. Measurements with horizontal excitations show a forced dynamic aperture of $(2.9 \pm 0.3) \sigma_{\text {nom }}$, and a free dynamic aperture of $(5.2 \pm$ $0.2) \sigma_{\text {nom }}$ for free oscillations.

Both results in the vertical and horizontal planes show a reduced forced dynamic aperture compared to the free 
dynamic aperture. This is of particular significance for top-energy applications in the LHC and High Luminosity LHC where ac dipole measurements are central to all beam optics commissioning strategies, and are limited in excitation amplitudes by the forced dynamic aperture. Forced dynamic aperture optimization is therefore crucial to allow optics measurements in highly nonlinear machines such as the High Luminosity LHC.

Multiparticle simulations show that the nominal model used with nonlinear magnetic errors fails to reproduce the measured forced dynamic aperture. The introduction of measured geometrical rotational errors and matching the coupling to the measured experimental values is needed in the model to reproduce the measured forced dynamic aperture. It demonstrates the usefulness of forced dynamic aperture measurements to characterize the nonlinear model.

The results presented in this paper demonstrate the potential of forced dynamic aperture as a new observable to provide insight on the nonlinear content of the machine, validate nonlinear corrections, improve the nonlinear model, and provide qualitative estimates of a lower bound for the free dynamic aperture.

\section{ACKNOWLEDGMENTS}

Particular thanks go to Gianluigi Arduini and Massimo Giovannozzi for proofreading the manuscript, and the Optics Measurements and Corrections team for providing useful and valuable insights.

[1] M. Bai et al., Overcoming Intrinsic Spin Resonances with an rf Dipole, Phys. Rev. Lett. 80, 4673 (1998).

[2] S. Peggs and C. Tang, Nonlinear diagnostics using an ac dipole, Brookhaven National Laboratory Technical Report No. RHIC/AP/159, 1998.

[3] M. Bai, S. Peggs, T. Roser, T. Satogata, and D. Trbojevic, Measuring beta function and phase advance in RHIC with an ac dipole, in Proceedings of the 2003 Particle Accelerator Conference, Portland, OR (IEEE, New York, 2003).

[4] X. Shen, S. Y. Lee, M. Bai, S. White, G. RobertDemolaize, Y. Luo, A. Marusic, and R. Tomás, Application of independent component analysis to ac dipole based optics measurement and correction at the relativistic heavy ion collider, Phys. Rev. ST Accel. Beams 16, 111001 (2013).

[5] R. Miyamoto, S. E. Kopp, A. Jannson, and M. J. Syphers, Tevatron ac dipole system, in Proceedings of the 22nd Particle Accelerator Conference, PAC-2007, Albuquerque, NM (IEEE, New York, 2007).

[6] R. Miyamoto, Diagnostics of the Fermilab Tevatron using an ac dipole, Ph.D. thesis, University of Texas, Austin, 2008.

[7] R. Miyamoto, S. E. Kopp, A. Jansson, and M. J. Syphers, Parametrization of the driven betatron oscillation, Phys. Rev. ST Accel. Beams 11, 084002 (2008).
[8] E. Carlier, L. Ducimetiere, and E. Vossenberg, A kicker pulse generator for measurement of the tune and dynamic aperture in the LHC, in Power Modulator Symposium, 2006. Conference Record of the 2006 Twenty-Seventh International (IEEE International Power Modulator Conference, Washington, DC, USA, 2006), pp. 463-466.

[9] R. A. Barlow, E. Carlier, J. P. Pianfetti, V. Senaj, and M. Cattin, Control of the MKQA tuning and aperture kickers of the LHC, CERN Technical Report No. CERN-TE-Note2010-001, 2009.

[10] R. Miyamoto, M. Cattin, J. Serrano, and R. Tomás, Signal quality of the LHC AC Dipoles and its impact on beam dynamics, CERN Technical Report No. CERN-ATS-2010063, 2010.

[11] J. Serrano and M. Cattin, The LHC AC Dipole system: An introduction, CERN Technical report, 2010.

[12] R. Tomás, T. Bach, R. Calaga, A. Langner, Y. I. Levinsen, E. H. Maclean, T. H. B. Persson, P. K. Skowronski, M. Strzelczyk, G. Vanbavinckhove, and R. Miyamoto, Record low $\beta$ beating in the LHC, Phys. Rev. ST Accel. Beams 15, 091001 (2012).

[13] R. Tomás, O. Brüning, M. Giovannozzi, P. Hagen, M. Lamont, F. Schmidt, G. Vanbavinckhove, M. Aiba, R. Calaga, and R. Miyamoto, CERN Large Hadron Collider optics model, measurements, and corrections, Phys. Rev. ST Accel. Beams 13, 121004 (2010).

[14] T. Persson, F. Carlier, J. Coello de Portugal, A. GarciaTabares Valdivieso, A. Langner, E. H. Maclean, L. Malina, P. Skowronski, B. Salvant, R. Tomás, and A. C. García Bonilla, LHC optics commissioning: A journey towards 1\% optics control, Phys. Rev. Accel. Beams 20, 061002 (2017).

[15] S. White, E. Maclean, and R. Tomás, Direct amplitude detuning measurement with ac dipole, Phys. Rev. ST Accel. Beams 16, 071002 (2013).

[16] E. H. Maclean, R. Tomás, M. Giovannozzi, and T. H. B. Persson, First measurement and correction of nonlinear errors in the experimental insertions of the CERN large hadron collider, Phys. Rev. ST Accel. Beams 18, 121002 (2015).

[17] R. Tomás, Normal form of particle motion under the influence of an ac dipole, Phys. Rev. ST Accel. Beams 5, 054001 (2002).

[18] F. S. Carlier, J. M. Coello De Portugal Martinez Vazquez, S. Fartoukh, E. Fol, D. Gamba, A. Garcia-Tabares Valdivieso, M. Giovannozzi, M. Hofer, A. S. Langner, E. H. Maclean, L. Malina, L. E. Medina Medrano, T. H. B. Persson, P. K. Skowronski, R. Tomás, F. Van Der Veken, and A. Wegscheider, Optics measurements and correction challenges for the HL-LHC, CERN Technical Report No. CERN-ACC-2017-0088, 2017.

[19] N. Fuster Martinez, E. H. Maclean, J. W. Dilly, L. J. Nevay, R. Bruce, R. Tomás, S. Redaelli, and T.H. B. Persson, Aperture measurements with AC dipole at the Large Hadron Collider, in Proceedings of International Particle Accelerator Conference (IPAC'18), Vancouver, Canada, 2018 (JACoW, Geneva, Switzerland, 2018), https://doi.org/ 10.18429/JACoW-IPAC2018-MOPMF048.

[20] R. Tomás, X. Buffat, S. White, J. Barranco, P. Gonçalves Jorge, and T. Pieloni, Beam-beam amplitude detuning with 
forced oscillations, Phys. Rev. Accel. Beams 20, 101002 (2017).

[21] N. Biancacci and R. Tomás, Using ac dipoles to localize sources of beam coupling impedance, Phys. Rev. Accel. Beams 19, 054001 (2016).

[22] A. Franchi, Recent measurements of linear and nonlinear optics at the ESRF storage ring, in 7th Low-Emittance Rings Workshop (2018), https://indico.cern.ch/event/ 671745/contributions/2788842/.

[23] I. Agapov, M. Bieler, H. Ehrlichmann, J. Keil, J. Klute, G. Kube, G. Sahoo, F. Schmidt-Fhre, R. Toms, R. Wanzenberg, and A. Wegscheider, Linear and nonlinear optics measurements with multiturn data at PETRA III, CERN Technical Report No. CERN-ACC-2017-258, 2017.

[24] U. Iriso (private communication).

[25] K. Hock, H. Huang, F. Méot, P. Oddo, N. Tsoupas, J. E. Tuozzolo, and K. Zeno, Status of ac-dipole project at RHIC injectors for polarized helions, in Proceedings of International Particle Accelerator Conference (IPAC'18), Vancouver, Canada, 2018 (JACoW, Geneva, Switzerland, 2018), https://doi.org/10.18429/JACoW-IPAC2018-TUPAF 005.

[26] S. Mönig, J. M. Coello de Portugal, A. Langner, E. Maclean, T. Persson, and R. Tomás, Short term dynamic aperture with ac dipoles, CERN Technical Report No. CERN-ACC-2016-173, 2016.

[27] W. Scandale, Dynamic aperture, CERN Technical Report No. CERN-SL-94-24-AP, 1994.

[28] A. Bazzani, M. Giovannozzi, G. Servizi, G. Turchetti, and E. Todesco, Normal forms for area preserving maps, resonances and dynamic aperture, CERN Technical Report No. CERN-SPS-89-24-AMS, 1989.

[29] M. Giovannozzi, Dynamic aperture for single-particle motion: Overview of theoretical background, numerical predictions and experimental results, AIP Conf. Proc. 693, 26 (2004).

[30] M Giovannozzi, A proposed scaling law for intensity evolution in hadron storage rings based on dynamic aperture variation with time, Phys. Rev. ST Accel. Beams 15, 024001 (2012).

[31] K. Ohmi, R. Tomás, Y. Funakoshi, R. Calaga, T. Ieiri, Y. Morita, K. Nakanishi, K. Oide, Y. Ohnishi, Y. Sun, M. Tobiyama, and F. Zimmermann, Response of colliding beam-beam system to harmonic excitation due to crabcavity rf phase modulation, Phys. Rev. ST Accel. Beams 14, 111003 (2011).

[32] MAD-X: Methodical accelerator design, http://madx.web .cern.ch/madx/.

[33] A. Franchi, L. Farvacque, F. Ewald, G. Le Bec, and K. B. Scheidt, First simultaneous measurement of sextupolar and octupolar resonance driving terms in a circular accelerator from turn-by-turn beam position monitor data, Phys. Rev. ST Accel. Beams 17, 074001 (2014).

[34] P. Hagen, M. Giovannozzi, J.-P. Koutchouk, T. Risselada, S. Sanfilippo, E. Todesco, and E. Wildner, WISE: An adaptative simulation of the LHC optics, CERN Technical Reports No. LHC-PROJECT-Report-971 and No. CERNLHC-Project-Report-971, 2006.

[35] G. Guignard, Betatron coupling and related impact of radiation, Phys. Rev. E 51, 6104 (1995).

[36] R Bartolini and F Schmidt, A computer code for frequency analysis of nonlinear betatron motion, CERN Technical Report No. SL-Note-98-017-AP, 1998.

[37] L. R. Carver et al., Instabilities and beam induced heating in 2016, in Proceedings Evian Workshop 16 (2016), https://cds.cern.ch/record/2293523?ln=en.

[38] V. Kiselev, E. Levichev, V. Sajaev, and V. Smaluk, Dynamic aperture study at the VEPP-4M Storage Ring, in Proceedings of the Particle Accelerator Conference, Vancouver, BC, Canada, 1997 (IEEE, New York, 1997).

[39] F. Willeke, Dynamic aperture, theoretical aspects, and observational features, in Beam measurement, Proceedings, Joint US-CERN-Japan-Russia School on Particle Accelerators, Montreux and Geneva, Switzerland, 1998, p. 326-350, 1998, https://cds.cern.ch/record/347310/.

[40] H. Maclean, E., R. Tomás, F. Schmidt, and T. H. B. Persson, Measurement of nonlinear observables in the Large Hadron Collider using kicked beams, Phys. Rev. ST Accel. Beams 17, 081002 (2014).

[41] D. Belohrad, L. K. Jensen, O. R. Jones, M. Ludwig, and J. J. Savioz, The LHC Fast BCT system: A comparison of design parameters with initial performance, CERN Technical Report CERN-BE-2010-010, 2010.

[42] S. Y. Lee, Accelerator Physics, 3rd ed. (World Scientific, Singapore, 2012). 